\title{
Hausdorff dimension and a generalized form of simultaneous Diophantine approximation
}

\author{
by
}

B. P. Rynne (Edinburgh) and H. Dickinson (York)

1. Introduction. Suppose that $m$ and $n$ are positive integers, $\boldsymbol{\tau}=$ $\left(\tau_{1}, \ldots, \tau_{m}\right) \in \mathbb{R}_{+}^{m}$ is a vector of strictly positive numbers, and $Q \subset \mathbb{Z}^{n}$ is an infinite set of integer vectors. Let $X$ denote a general point in $\mathbb{R}^{m n}$, which we will write in the form $X=\left(\mathbf{x}_{1}, \ldots, \mathbf{x}_{m}\right)$, with $\mathbf{x}_{i} \in \mathbb{R}^{n}, i=1, \ldots, m$, and define the set

$$
\begin{aligned}
& W_{Q}(m, n ; \boldsymbol{\tau}) \\
& \quad=\left\{X \in \mathbb{R}^{m n}:\left\|\mathbf{x}_{i} \cdot \mathbf{q}\right\|<|\mathbf{q}|^{-\tau_{i}}, 1 \leq i \leq m, \text { for infinitely many } \mathbf{q} \in Q\right\}
\end{aligned}
$$

(where, for any $z \in \mathbb{R},\|z\|$ denotes the distance from $z$ to the nearest integer). In the special case $\boldsymbol{\tau}=\boldsymbol{\tau}(\tau)=(\tau, \ldots, \tau)$, for $\tau>0$, and $Q=\mathbb{Z}^{n}$, the set $W_{\mathbb{Z}^{n}}(m, n ; \boldsymbol{\tau}(\tau))$ has been studied by many authors; in particular, its Hausdorff dimension has been obtained. Jarník [8] and Besicovitch [1] showed that if $\tau>1$, then $\operatorname{dim} W_{\mathbb{Z}}(1,1 ; \boldsymbol{\tau}(\tau))=2 /(1+\tau)$ (dim denotes Hausdorff dimension). Later Jarník [9] and Eggleston [7] showed that if $\tau>1 / m$, then $\operatorname{dim} W_{\mathbb{Z}}(m, 1 ; \boldsymbol{\tau}(\tau))=(m+1) /(1+\tau)$. Furthermore, Eggleston obtained the dimension of $W_{Q}(m, 1 ; \boldsymbol{\tau}(\tau))$ for certain infinite sets $Q \subset \mathbb{Z}$ and Bovey and Dodson [3] obtained the dimension of $W_{Q}(m, n ; \boldsymbol{\tau}(\tau))$ for certain $Q \in \mathbb{Z}^{n}$. These results were extended to arbitrary infinite sets $Q \subset \mathbb{Z}$ by Borosh and Fraenkel [2] and to arbitrary $Q \subset \mathbb{Z}^{n}$ by Rynne [10].

To state their results we need the following definition. Suppose that $Q \subset \mathbb{Z}^{n}$ is an arbitrary infinite set and let

$$
\nu(Q)=\inf \left\{\nu \in \mathbb{R}: \sum_{\mathbf{q} \in Q}|\mathbf{q}|^{-\nu}<\infty\right\} .
$$

Clearly, $0 \leq \nu(Q) \leq n$. It is shown in [10] that if $\tau \geq \nu(Q) / m$, then

$$
\operatorname{dim} W_{Q}(m, n ; \boldsymbol{\tau}(\tau))=m(n-1)+\frac{m+\nu(Q)}{1+\tau} .
$$

2000 Mathematics Subject Classification: Primary 11J83, 28A78. 
This result was extended in [11] to the set $W_{Q}(m, 1 ; \boldsymbol{\tau})$ for general $\boldsymbol{\tau}$. Such an extension also exists for $m=2$ and $n=1$ for the simultaneous approximation of real numbers by algebraic numbers of bounded degree [6]. In the present paper we will obtain the Hausdorff dimension of $W_{Q}(m, n ; \boldsymbol{\tau})$ for general $n$.

Without loss of generality we will suppose throughout that $\tau_{1} \geq \ldots$ $\geq \tau_{m}$. Let $\sigma(\boldsymbol{\tau})=\sum_{i=1}^{m} \tau_{i}$, and define the number

$$
D_{Q}(m, n ; \boldsymbol{\tau})=m(n-1)+\min _{1 \leq k \leq m}\left\{\frac{m+\nu(Q)+\sum_{i=k}^{m}\left(\tau_{k}-\tau_{i}\right)}{1+\tau_{k}}\right\} .
$$

Theorem 1.1. If $\sigma(\boldsymbol{\tau}) \geq \nu(Q)$, then

$$
\operatorname{dim} W_{Q}(m, n ; \boldsymbol{\tau})=D_{Q}(m, n ; \boldsymbol{\tau}) .
$$

If $\sigma(\boldsymbol{\tau}) \leq \nu(Q)$, then $\operatorname{dim} W_{Q}(m, n ; \boldsymbol{\tau})=m n$.

REMARK 1.2. It will be shown at the end of the proof of Theorem 1.1 that if $\sigma(\boldsymbol{\tau})=\nu(Q)$ then $D_{Q}(m, n ; \boldsymbol{\tau})=m n$ so the results in the two cases in the theorem are consistent.

The above problem can be generalized in the manner considered in [4]. Let $\boldsymbol{\psi}=\left(\psi_{1}, \ldots, \psi_{m}\right)$ be a collection of non-negative functions on $\mathbb{Z}^{n}$ (the functions $\psi_{i}$ need only be defined on $Q$, but for simplicity we ignore this). Now define the set

$$
\begin{aligned}
& W_{Q}(m, n ; \boldsymbol{\psi}) \\
& \quad=\left\{X \in \mathbb{R}^{m n}:\left\|\mathbf{x}_{i} \cdot \mathbf{q}\right\|<\psi_{i}(\mathbf{q}), 1 \leq i \leq m, \text { for infinitely many } \mathbf{q} \in Q\right\}
\end{aligned}
$$

Under a further assumption on the limiting behaviour of the functions $\psi_{i}$ we can obtain the dimension of $W_{Q}(m, n ; \boldsymbol{\psi})$. Suppose that the limits

$$
\lambda\left(\psi_{i}\right)=\lim _{|\mathbf{q}| \rightarrow \infty} \frac{-\log \psi_{i}(\mathbf{q})}{\log |\mathbf{q}|}, \quad i=1, \ldots, m,
$$

exist and are positive, and put $\boldsymbol{\tau}(\boldsymbol{\psi}):=\left(\lambda\left(\psi_{1}\right), \ldots, \lambda\left(\psi_{m}\right)\right)$. Then from Theorem 1.1 we obtain the following result.

Corollary 1.3. If $\sigma(\boldsymbol{\tau}(\boldsymbol{\psi})) \geq \nu(Q)$, then

$$
\operatorname{dim} W_{Q}(m, n ; \boldsymbol{\psi})=D_{Q}(m, n ; \boldsymbol{\tau}(\boldsymbol{\psi})) .
$$

If $\sigma(\boldsymbol{\tau}(\boldsymbol{\psi})) \leq \nu(Q)$, then $\operatorname{dim} W_{Q}(m, n ; \boldsymbol{\psi})=m n$.

Proof. From the hypotheses on the functions $\psi_{i}$ we have, for any $\varepsilon>0$ and each $i=1, \ldots, m$,

$$
|\mathbf{q}|^{-\lambda\left(\psi_{i}\right)-\varepsilon} \leq \psi_{i}(\mathbf{q}) \leq|\mathbf{q}|^{-\lambda\left(\psi_{i}\right)+\varepsilon},
$$

for all sufficiently large $|\mathbf{q}| \in Q$. Thus, letting $\varepsilon=(\varepsilon, \ldots, \varepsilon)$, it follows that

$$
W_{Q}(m, n ; \boldsymbol{\tau}(\boldsymbol{\psi})+\boldsymbol{\varepsilon}) \subset W_{Q}(m, n ; \boldsymbol{\psi}) \subset W_{Q}(m, n ; \boldsymbol{\tau}(\boldsymbol{\psi})-\boldsymbol{\varepsilon}) .
$$


Now, letting $\varepsilon \rightarrow 0$, the result follows from these inclusions and the continuity with respect to $\tau$ of the dimension result in Theorem 1.1 (see Remark 1.2).

2. Proof of Theorem 1.1. To fix our notation we first recall the (standard) definition of the Hausdorff dimension of an arbitrary set $E \subset \mathbb{R}^{r}$, for any positive integer $r$. Let $\mathcal{I}$ be a countable collection of bounded sets $I \subset \mathbb{R}^{r}$. For any $\varrho>0$, the $\varrho$-volume of the collection $\mathcal{I}$ is defined to be

$$
V_{\varrho}(\mathcal{I})=\sum_{I \in \mathcal{I}} d(I)^{\varrho}
$$

where $d(I)=\sup \left\{|\mathbf{x}-\mathbf{y}|_{2}: \mathbf{x}, \mathbf{y} \in I\right\}$ is the diameter of $I$ and $|\cdot|_{2}$ denotes the usual Euclidean norm in $\mathbb{R}^{r}$. For every $\eta>0$ define

$$
m_{\varrho}(\eta, E)=\inf V_{\varrho}(\mathcal{I})
$$

where the infimum is taken over all countable collections, $\mathcal{I}$, of sets $I$ with diameter $d(I) \leq \eta$, that cover $E$. Now define the $\varrho$-dimensional Hausdorff outer measure of $E$ to be

$$
m_{\varrho}(E)=\sup _{\eta>0} m_{\varrho}(\eta, E) .
$$

The Hausdorff dimension of $E$ is defined to be

$$
\operatorname{dim} E=\inf \left\{\varrho: m_{\varrho}(E)=0\right\} .
$$

We also require some further notation. For any finite set $A$ we let $|A|$ denote the cardinality of $A$. The notation $a \ll b$ (respectively $a \gg b$ ) will denote an inequality of the form $a \leq c b$ (respectively $a \geq c b$ ), where $c>0$ is a constant which depends at most on $m, n, \nu(Q), \tau$ and $\delta$ (which will be introduced below); similarly, $c_{1}, c_{2}, \ldots$ will denote positive constants which depend at most on $m, n, \nu(Q), \tau$ and $\delta$. If $a \ll b \ll a$ then we write $a \approx b$. A set of the form $B=\left\{\mathbf{x} \in \mathbb{R}^{r}:|\mathbf{x}-\mathbf{b}|_{2} \leq d / 2\right\}$, for any $r \geq 1$, is said to be a ball of diameter $d$ and centre $\mathbf{b}$. If $\alpha>0$ is a real number then $\alpha B$ will denote the ball with centre $\mathbf{b}$ and diameter $\alpha d$. Let $U_{n}$ denote the unit cube

$$
U_{n}=\left\{\mathbf{x} \in \mathbb{R}^{n}: 0 \leq x_{i} \leq 1, i=1, \ldots, n\right\},
$$

and let $U\left(=U_{m n}\right)$ be the Cartesian product $U=\times_{i=1}^{m} U_{n} \subset \mathbb{R}^{m n}$.

We can now begin the proof of the theorem. Since $W_{Q}(m, n ; \boldsymbol{\tau})$ is invariant under translations by integer vectors it suffices to consider the set $W_{Q}(m, n ; \boldsymbol{\tau}) \cap U$. The proof is in two parts - we obtain, separately, an upper bound and a lower bound for $\operatorname{dim} W_{Q}(m, n ; \boldsymbol{\tau}) \cap U$. The proof of the upper bound $\operatorname{dim} W_{Q}(m, n ; \boldsymbol{\tau}) \cap U \leq D_{Q}(m, n ; \boldsymbol{\tau})$, for $\sigma(\boldsymbol{\tau}) \geq \nu(Q)$, is relatively straightforward and follows from combining the corresponding arguments in [10] and in [11] (the bound $\operatorname{dim} W_{Q}(m, n ; \boldsymbol{\tau}) \leq m n$ is trivial). For brevity we will omit the details. 
To prove the reverse inequality for $\operatorname{dim} W_{Q}(m, n ; \boldsymbol{\tau}) \cap U$ we first require some lemmas. Suppose, for now, that $\nu=\nu(Q)>0$ and $\sigma(\boldsymbol{\tau})>\nu$, and let $\delta>0$ be an arbitrarily small number satisfying

$$
0<\delta<\min \{\nu, \sigma(\boldsymbol{\tau})-\nu, 1\}
$$

(the cases where the above assumptions do not hold will be dealt with at the end of the proof). Some other restrictions will be imposed on $\delta$ below, but essentially $\delta$ is a fixed "sufficiently small" number. Since the case $n=1$ was dealt with in [11] we will also suppose that $n \geq 2$.

We also suppose that the series $\sum_{\mathbf{q} \in Q}|\mathbf{q}|^{-\nu}$ is divergent. If this assumption does not hold we replace $\nu$ with $\nu-\varepsilon, \varepsilon>0$, throughout the following argument to obtain

$$
\operatorname{dim} W_{Q}(m, n ; \boldsymbol{\tau}) \geq m(n-1)+\min _{1 \leq k \leq m}\left\{\frac{m+\nu-\varepsilon+\sum_{i=k}^{m}\left(\tau_{k}-\tau_{i}\right)}{1+\tau_{k}}\right\},
$$

which yields the result since $\varepsilon>0$ is arbitrary.

Lemma 2.1 (Lemma 2.1 of [10]). For any integer $k_{0}>0$ there exists an integer $k>k_{0}$ such that

$$
\sum_{\substack{\mathbf{q} \in Q \\ 2^{k} \leq|\mathbf{q}|<2^{k+1}}} 1 \geq 2^{k \nu} / k^{2} .
$$

From now on, $N$ will always denote an integer of the form $2^{k}$, where $k$ is such that (2) holds. By Lemma 2.1 there are infinitely many such integers. Thus, writing

$$
Q(N)=\{\mathbf{q} \in Q: N \leq|\mathbf{q}|<2 N\},
$$

we have

$$
|Q(N)| \geq N^{\nu-\delta / 2}
$$

for all sufficiently large $N$ (of the above form). Now, for any vector $\mathbf{q} \in$ $Q(N)$, let $[\mathbf{q}] \subset Q$ denote the set of all those vectors $\mathbf{q}^{\prime} \in Q(N)$ which are linearly dependent on q. Clearly the relation of linear dependence is an equivalence relation on the set $Q(N)$ and we let $[Q(N)]$ denote the corresponding set of equivalence classes $[\mathbf{q}]$.

Lemma 2.2 (Lemma 2.2 of [10]). There exists a number $\alpha$, with $\delta \leq \alpha \leq$ $\nu$, and a subset $\widetilde{Q} \subset Q$ such that, for infinitely many $N$,

$$
\begin{aligned}
|[\widetilde{Q}(N)]| & \approx N^{\alpha-\delta}, \\
|[\mathbf{q}]| & \approx N^{\nu-\alpha},
\end{aligned}
$$

for all equivalence classes $[\mathbf{q}] \in[\widetilde{Q}(N)]$. Thus

$$
|\widetilde{Q}(N)| \approx N^{\nu-\delta} \text {. }
$$


It should be noted that the number $\alpha$ here was denoted by $\gamma$ in [10]. We now suppose that $\nu-\alpha>0$. The case where this does not hold will be discussed at the end of the proof.

Lemma 2.3 (Lemma 1 of [11]). The following result holds for almost all collections in the set $\left\{\boldsymbol{\tau} \in \mathbb{R}_{+}^{m}: \sigma(\boldsymbol{\tau}) \geq \nu\right\}$ (here, "almost all" is with respect to Lebesgue measure in $\left.\mathbb{R}^{m}\right)$. There exists an integer $K=K(\boldsymbol{\tau}), 1 \leq K \leq m$, and a number $\delta_{0}=\delta_{0}(\boldsymbol{\tau})>0$ such that for any $\delta \in\left(0, \delta_{0}\right)$ there exists a collection of numbers $\widetilde{\boldsymbol{\tau}}=\widetilde{\boldsymbol{\tau}}(\delta)=\left(\widetilde{\tau}_{1}(\delta), \ldots, \widetilde{\tau}_{m}(\delta)\right) \in \mathbb{R}_{+}^{m}$, with the following properties:

$(\tau 1) \tau_{i}-\delta / m=\widetilde{\tau}_{i} \geq \tau_{i+1}+\delta / m$ for each $i=K+1, \ldots, m ;$

$(\tau 2) \tau_{K}-2 \delta / m \geq \widetilde{\tau}_{1}=\ldots=\widetilde{\tau}_{K} \geq \tau_{K+1}+\delta / m$

$(\tau 3) \sum_{i=1}^{m} \widetilde{\tau}_{i}=\nu$.

In particular, $\widetilde{\tau}_{1} \geq \ldots \geq \widetilde{\tau}_{m}$.

REMARK 2.4. If $K=m$ then condition $(\tau 1)$ and the second inequality in condition $(\tau 2)$ are to be ignored. We adopt the convention that any arguments relating to situations which cannot occur for a particular choice of numbers are to be ignored in that particular case.

Let $G$ denote the set of collections $\tau$ for which the conclusions of Lemma 2.3 hold. By the continuity argument following the proof of Lemma 1 in [11], we need only prove the required lower bound for $\operatorname{dim} W_{Q}(m, n ; \boldsymbol{\tau})$ for all $\boldsymbol{\tau} \in G$. Thus from now on we consider a fixed $\boldsymbol{\tau} \in G$ and write $\sigma$ for $\sigma(\boldsymbol{\tau})$.

We now require some further notation. For any $\mathbf{q} \in \mathbb{Z}^{n}, t \in \mathbb{Z}$, let $H(\mathbf{q}, t) \subset \mathbb{R}^{n}$ denote the $(n-1)$-dimensional hyperplane $\left\{\mathbf{x} \in \mathbb{R}^{n}: \mathbf{x} \cdot \mathbf{q}+t=\right.$ $0\}$. If $\mathbf{t} \in \mathbb{Z}^{m}$, let $H(\mathbf{q}, \mathbf{t})=\mathbf{X}_{i=1}^{m} H\left(\mathbf{q}, t_{i}\right) \subset \mathbb{R}^{m n}$. The next lemma is an adaptation of Lemma 4 in [2], Lemma 2.3 of [10] and Lemma 2 of [11].

Lemma 2.5. For any number $L$ with $0<L<1$, there exist arbitrarily large integers $N$ such that, for every ball $C \subset U$ with diameter $L$, and every equivalence class $[\mathbf{q}] \in[\widetilde{Q}(N)]$, there is a set $S=S(C,[\mathbf{q}])$, consisting of pairs $(\mathbf{q}, \mathbf{t}), \mathbf{q} \in[\mathbf{q}]$ and $\mathbf{t} \in \mathbb{Z}^{m}$, with the properties:

(i) for all $(\mathbf{q}, \mathbf{t}) \in S, H(\mathbf{q}, \mathbf{t}) \cap \frac{1}{2} C \neq \emptyset$, which

(ii) for all distinct pairs $\left(\mathbf{q}^{1}, \mathbf{t}^{1}\right),\left(\mathbf{q}^{2}, \mathbf{t}^{2}\right) \in S$, there is an integer $i$ for

$$
\left|H\left(\mathbf{q}^{1}, t_{i}^{1}\right)-H\left(\mathbf{q}^{2}, t_{i}^{2}\right)\right|_{2} \geq c_{1} N^{-1-\widetilde{\tau}_{i}+\alpha / m-\delta / m} ;
$$

(iii) the number of pairs $(\mathbf{q}, \mathbf{t})$ in $S$ satisfies

$$
|S| \gg L^{m} \chi([\mathbf{q}]) \gg L^{m} N^{m+\nu-\alpha-\delta / 2},
$$

where $\chi([\mathbf{q}])=\sum_{\mathbf{q} \in[\mathbf{q}]} \phi(|\mathbf{q}|)^{m}$ and $\phi$ is the Euler function; 
(iv) for any set $I \subset C$ with $d(I)>N^{-1+\delta}$, let $S_{I}$ denote the set of pairs $(\mathbf{q}, \mathbf{t}) \in S$ for which $H(\mathbf{q}, \mathbf{t}) \cap I \neq \emptyset$. Then

$$
\left|S_{I}\right| \ll d(I)^{m} \chi([\mathbf{q}]) .
$$

Pr o of. The proof of Lemma 2.3 in [10] is based on the results in Lemma 4 of [2]. The present lemma can be proved in a similar manner, but based on the results in Lemma 2 of [11] (which in turn was based on the proof of Lemma 4 in [2]). We will omit the details.

We now suppose that $L$ and $C \subset U$, with $d(C)=L$, are fixed, and choose $N$ so that Lemma 2.5 holds. We now wish to construct a collection of balls in $C$ lying "close" to the planes $H(\mathbf{q}, \mathbf{t}),(\mathbf{q}, \mathbf{t}) \in S([\mathbf{q}])$, where $S([\mathbf{q}])$ is the set constructed in Lemma 2.5 (to simplify the notation slightly we have suppressed the dependence of $S$ on $C$ ). To ensure that the balls from different such collections do not intersect we need the following rather complicated construction.

For any equivalence class $[\mathbf{q}] \in[\widetilde{Q}(N)]$ let

$$
E([\mathbf{q}])=\bigcup_{(\mathbf{q}, \mathbf{t}) \in S([\mathbf{q}])}\left(H(\mathbf{q}, \mathbf{t}) \cap \frac{3}{4} C\right) .
$$

Since the planes $H(\mathbf{q}, \mathbf{t})$, with $(\mathbf{q}, \mathbf{t}) \in S([\mathbf{q}])$, pass through the ball $\frac{1}{2} C$, the $m(n-1)$-dimensional Lebesgue measure (which we denote by $\left.\mu_{m(n-1)}\right)$ of the set $H(\mathbf{q}, \mathbf{t}) \cap \frac{3}{4} C$ satisfies $\mu_{m(n-1)}\left(H(\mathbf{q}, \mathbf{t}) \cap \frac{3}{4} C\right) \gg L^{m(n-1)}$, and hence by $(7)$,

$$
\mu_{m(n-1)}(E([\mathbf{q}])) \gg L^{m n} \chi([\mathbf{q}]) \gg L^{m n} N^{m+\nu-\alpha-\delta} .
$$

Now, for any $\mathbf{p} \in \widetilde{Q}(N), \mathbf{p} \notin[\mathbf{q}]$ and any pair $(\mathbf{q}, \mathbf{t}) \in S([\mathbf{q}])$, let

$$
F(\mathbf{p} ; \mathbf{q}, \mathbf{t})=\left\{X \in H(\mathbf{q}, \mathbf{t}) \cap \frac{3}{4} C:\left\|\mathbf{x}_{i} \cdot \mathbf{p}\right\|<8 n N^{-\widetilde{\tau}_{i}-\delta / m}, i=1, \ldots, m\right\} .
$$

Let

$$
F([\mathbf{q}])=\bigcup_{\substack{\mathbf{p} \in \widetilde{Q}(N) \\ \mathbf{p} \notin[\mathbf{q}]}} \bigcup_{(\mathbf{q}, \mathbf{t}) \in S([\mathbf{q}])} F(\mathbf{p} ; \mathbf{q}, \mathbf{t}) .
$$

Lemma 2.6 (Lemma 2.4 of $[10]$ ). For any $[\mathbf{q}] \in \widetilde{Q}(N)$,

$$
\frac{\mu_{m(n-1)}(F([\mathbf{q}]))}{\mu_{m(n-1)}(E([\mathbf{q}]))} \ll L^{-m n} N^{-\delta} .
$$

Proof. For any $\mathbf{p} \neq \mathbf{0}$ and any $\eta \geq 0$, let

$$
A_{\mathbf{p}}(\eta)=\left\{\mathbf{x} \in U_{n}:\|\mathbf{x} \cdot \mathbf{p}\| \leq \eta\right\} .
$$

It is shown in [5] or [12] that if $\mathbf{p}$ and $\mathbf{p}^{\prime}$ are linearly independent integer vectors then, for any $\eta, \eta^{\prime}>0$,

$$
\mu_{n}\left(A_{\mathbf{p}}(\eta) \cap A_{\mathbf{p}^{\prime}}\left(\eta^{\prime}\right)\right)=4 \eta \eta^{\prime} .
$$


Now, by definition,

$$
F([\mathbf{q}]) \subset \bigcup_{\substack{\mathbf{p} \in \widetilde{Q}(N) \\ \mathbf{p} \notin[\mathbf{q}]}} \bigcup_{\mathbf{q} \in[\mathbf{q}]} \underset{i=1}{\times}\left(A_{\mathbf{p}}\left(N^{-\widetilde{\tau}_{i}-\delta / m}\right) \cap A_{\mathbf{q}}(0)\right),
$$

so

$$
\mu_{m(n-1)}(F([\mathbf{q}])) \leq \sum_{\substack{\mathbf{p} \in \widetilde{Q}(N) \\ \mathbf{p} \notin[\mathbf{q}]}} \sum_{\mathbf{q} \in[\mathbf{q}]} \prod_{i=1}^{m} \mu_{n-1}\left(A_{\mathbf{p}}\left(N^{-\widetilde{\tau}_{i}-\delta / m}\right) \cap A_{\mathbf{q}}(0)\right) .
$$

For each $\eta>0$, the set $A_{\mathbf{p}}\left(N^{-\widetilde{\tau}_{i}-\delta / m}\right) \cap A_{\mathbf{q}}(\eta)$ is an $n$-dimensional "thickening" of the set $A_{\mathbf{p}}\left(N^{-\widetilde{\tau}_{i}-\delta / m}\right) \cap A_{\mathbf{q}}(0)$ (which consists of portions of $(n-1)$ dimensional planes) with "thickness" $2 \eta|\mathbf{q}|_{2}^{-1}$. Thus

$$
\begin{aligned}
\mu_{n-1}\left(A_{\mathbf{p}}\left(N^{-\widetilde{\tau}_{i}-\delta / m}\right) \cap A_{\mathbf{q}}(0)\right) & =\lim _{\eta \rightarrow 0} \mu_{n}\left(A_{\mathbf{p}}\left(N^{-\widetilde{\tau}_{i}-\delta / m}\right) \cap A_{\mathbf{q}}(\eta)\right) / 2 \eta|\mathbf{q}|_{2}^{-1} \\
& \ll N^{1-\widetilde{\tau}_{i}-\delta / m},
\end{aligned}
$$

by (9). Hence by $(\tau 3),(4)$ and $(5)$,

$$
\begin{aligned}
\mu_{m(n-1)}(F([\mathbf{q}])) & \ll \sum_{\substack{\mathbf{p} \in \widetilde{Q}(N) \\
\mathbf{p} \notin[\mathbf{q}]}} \sum_{\mathbf{q} \in[\mathbf{q}]} \prod_{i=1}^{m} N^{1-\widetilde{\tau}_{i}-\delta / m} \\
& \ll N^{\nu-\delta} N^{\nu-\alpha} N^{m-\nu-\delta}=N^{m+\nu-\alpha-2 \delta},
\end{aligned}
$$

so the result follows from (8).

Now, it follows from Lemma 2.6 that for $N$ sufficiently large we can choose a collection $\mathcal{B}^{0}([\mathbf{q}])$ of pairwise disjoint balls $B \subset \frac{3}{4} C$, in $\mathbb{R}^{m n}$, with diameter $n^{-1}(2 N)^{-\left(1+\tau_{1}\right)}$, whose centres $Z$ lie on $E([\mathbf{q}]) \backslash F([\mathbf{q}])$, and satisfy

$$
\left|Z-Z^{\prime}\right|_{2} \geq 4 N^{-\left(1+\tau_{1}\right)} \quad \text { if } Z \neq Z^{\prime},
$$

and such that

$$
\left|\mathcal{B}^{0}([\mathbf{q}])\right| \gg \frac{\mu_{m(n-1)}(E([\mathbf{q}]))}{\left(N^{-\left(1+\tau_{1}\right)}\right)^{m(n-1)}} \gg L^{m n} \chi([\mathbf{q}]) N^{m(n-1)\left(1+\tau_{1}\right)}
$$

(by (8)). Since each $B \in \mathcal{B}^{0}([\mathbf{q}])$ has diameter $n^{-1}(2 N)^{-\left(1+\tau_{1}\right)}$, and lies on some plane $H(\mathbf{q}, \mathbf{t})$, with $\mathbf{q} \in[\mathbf{q}]$, it follows that if $X=\left(\mathbf{x}_{1}, \ldots, \mathbf{x}_{m}\right) \in B$ then for each $i=1, \ldots, m$,

$$
\left\|\mathbf{x}_{i} \cdot \mathbf{q}\right\| \leq n^{-1}(2 N)^{-\left(1+\tau_{1}\right)}|\mathbf{q}|_{2}<(2 N)^{-\tau_{1}} \leq|\mathbf{q}|^{-\tau_{i}}
$$

(using $|\mathbf{q}|_{2}<2 n N$ for all $\mathbf{q} \in \widetilde{Q}(N)$ ), so $B$ has the property:

(12) if $X \in B$ then there exists $\mathbf{q} \in[\mathbf{q}]$ such that $\left\|\mathbf{x}_{i} \cdot \mathbf{q}\right\|<|\mathbf{q}|^{-\tau_{i}}$, $i=1, \ldots, m$. 
Now choose an arbitrary ball $B^{0} \in \mathcal{B}^{0}([\mathbf{q}])$, with centre $Z^{0}=\left(\mathbf{z}_{1}^{0}, \ldots, \mathbf{z}_{m}^{0}\right)$. For each vector $\mathbf{r} \in \mathbb{Z}^{m}$, with

$$
r_{1}=0, \quad\left|r_{i}\right|<(8 n)^{-1} 2^{-\tau_{1}} N^{\tau_{1}-\tau_{i}}, \quad i=2, \ldots, m,
$$

let $B^{\mathbf{r}}\left(B^{0}\right)$ be the ball with diameter $n^{-1}(2 N)^{-\left(1+\tau_{1}\right)}$ and centre $Z^{\mathbf{r}}=$ $\left(\mathbf{z}_{1}^{\mathbf{r}}, \ldots, \mathbf{z}_{m}^{\mathbf{r}}\right)$, where

$$
\mathbf{z}_{i}^{\mathbf{r}}=\mathbf{z}_{i}^{0}+r_{i} 4 N^{-\left(1+\tau_{1}\right)} \mathbf{q} /|\mathbf{q}|_{2}, \quad i=1, \ldots, m,
$$

(note that the unit vector $\mathbf{q} /|\mathbf{q}|_{2}$ is orthogonal to the plane $H(\mathbf{q}, t)$ in $\mathbb{R}^{n}$, for any $t \in \mathbb{R})$. We let $\mathcal{B}\left(B^{0}\right)$ denote the collection $\mathcal{B}\left(B^{0}\right)=\bigcup_{\mathbf{r}} B^{\mathbf{r}}\left(B^{0}\right)$ (where the union is over all vectors $\mathbf{r}$ satisfying (13)). If $N$ is sufficiently large, then each ball $B \in \mathcal{B}\left(B^{0}\right)$ satisfies $B \subset C$ and property (12) (by a similar calculation to the above, using (13)). Furthermore, (if $c_{1} N^{\alpha / m} \geq 4$ ) from (6) and the above construction, if the balls $B^{1}, B^{2}$ in $\mathcal{B}^{0}([\mathbf{q}])$ lie on different planes $H(\mathbf{q}, t)$ then the centres $Z, Z^{\prime}$ of any two balls $B \in \mathcal{B}\left(B^{1}\right)$, $B^{\prime} \in \mathcal{B}\left(B^{2}\right)$, satisfy

$$
\left|\mathbf{z}_{i}-\mathbf{z}_{i}^{\prime}\right|_{2} \geq N^{-1-\widetilde{\tau}_{i}-\delta / m}, \quad \text { for some } i,
$$

(again using $|\mathbf{q}|_{2}<2 n N$ for all $\mathbf{q} \in \widetilde{Q}(N)$, and also $\tau_{1}-\delta / m \geq \widetilde{\tau}_{i}+\delta / m$ for all $i$ ).

Repeating this process for all $B^{0} \in \mathcal{B}^{0}([\mathbf{q}])$ we obtain the collection

$$
\mathcal{B}([\mathbf{q}])=\bigcup_{B^{0} \in \mathcal{B}^{0}([\mathbf{q}])} \mathcal{B}\left(B^{0}\right) .
$$

Each $B \in \mathcal{B}([\mathbf{q}])$ has the property (12), and it follows from (14) that all the balls in $\mathcal{B}([\mathbf{q}])$ are disjoint, and so, from (11) and the number of vectors $\mathbf{r}$ satisfying (13), we have

$$
\begin{aligned}
|\mathcal{B}([\mathbf{q}])| & \gg L^{m n} \chi([\mathbf{q}]) N^{m(n-1)\left(1+\tau_{1}\right)} \prod_{i=1}^{m} N^{\tau_{1}-\tau_{i}} \\
& \gg L^{m n} \chi([\mathbf{q}]) N^{m(n-1)\left(1+\tau_{1}\right)+\gamma}
\end{aligned}
$$

where $\gamma=\sum_{i=1}^{m}\left(\tau_{1}-\tau_{i}\right)=m \tau_{1}-\sigma$.

Repeating the above constructions for each $[\mathbf{q}] \in[\widetilde{Q}(N)]$ we obtain the collection

$$
\mathcal{B}=\bigcup_{[\mathbf{q}] \in[\widetilde{Q}(N)]} \mathcal{B}([\mathbf{q}])
$$

If $[\mathbf{q}] \neq\left[\mathbf{q}^{\prime}\right]$ and $B \in \mathcal{B}([\mathbf{q}]), B^{\prime} \in \mathcal{B}\left(\left[\mathbf{q}^{\prime}\right]\right)$ then it follows from the definition of the sets $F(\mathbf{p} ; \mathbf{q}, \mathbf{t})$ and the above construction that the centres of these balls, $Z$ and $Z^{\prime}$ respectively, satisfy (14). Hence, in particular, all the balls in the collection $\mathcal{B}$ are disjoint.

Using these constructions we can now prove the following lemma, which is similar to Lemmas 2.5 and 2.6 of [10], or Lemma 3 of [11]. For the reader's 
convenience we summarize here certain relationships between the various numbers we have introduced above:

$$
\nu=\sum_{i=1}^{m} \widetilde{\tau}_{i}, \quad \sigma=\sum_{i=1}^{m} \tau_{i}, \quad \gamma=\sum_{i=1}^{m}\left(\tau_{1}-\tau_{i}\right)=m \tau_{1}-\sigma .
$$

Lemma 2.7. For any number $L$ with $0<L<1$, there exist arbitrarily large integers $N$ such that for any ball $C \subset U$ with diameter $L$ there is a collection $\mathcal{B}$ of disjoint balls $B \subset C$, such that:

(i) each $B \in \mathcal{B}$ has diameter $n^{-1}(2 N)^{-\left(1+\tau_{1}\right)}$ and the centres of any two balls in $\mathcal{B}$ are at least a distance $4 N^{-\left(1+\tau_{1}\right)}$ apart;

(ii) for each $B \in \mathcal{B}$, (12) holds for some $[\mathbf{q}] \in[\widetilde{Q}(N)]$;

(iii) $|\mathcal{B}| \geq c_{2} L^{m n} X(N) N^{m(n-1)\left(1+\tau_{1}\right)+\gamma}$, where

$$
X(N)=\sum_{[\mathbf{q}] \in[\widetilde{Q}(N)]} \chi([\mathbf{q}]) \gg N^{m+\nu-3 \delta / 2}
$$

(iv) if $I$ is a set in $\mathbb{R}^{m n}$ with $d(I) \geq n^{-1} N^{-\left(1+\tau_{1}\right)}$, which intersects $h$ of the balls $B$ in $\mathcal{B}$, then:

(a) suppose that $N^{-\left(1+\tau_{k}\right)}<d(I) \leq N^{-\left(1+\tau_{k+1}\right)}$, for some $k$ with $1 \leq$ $k \leq m-1$ :

- if $k<K$, then

$$
h \leq c_{3} d(I)^{m n-k} N^{(m n-k)\left(1+\tau_{1}\right)+\sum_{i=1}^{k}\left(\tau_{1}-\tau_{i}\right)} ;
$$

- if $k=K$, then

$$
\begin{aligned}
h \leq & c_{3} d(I)^{m n-k} N^{(m n-k)\left(1+\tau_{1}\right)+\sum_{i=1}^{k}\left(\tau_{1}-\tau_{i}\right)} \\
& +c_{3} d(I)^{m n} N^{m(n-1)\left(1+\tau_{1}\right)+m+\nu+\gamma+\delta}
\end{aligned}
$$

- if $k>K$, then

$$
h \leq c_{3} d(I)^{m n} N^{m(n-1)\left(1+\tau_{1}\right)+m+\nu+\gamma+\delta} ;
$$

(b) if $N^{-\left(1+\tau_{m}\right)}<d(I) \leq N^{-1+\delta}$, then

$$
h \leq c_{3} d(I)^{m n} N^{m(n-1)\left(1+\tau_{1}\right)+m+\nu+\gamma}
$$

(c) if $N^{-1+\delta}<d(I)$, then

$$
h \leq c_{3} d(I)^{m n} X(N) N^{m(n-1)\left(1+\tau_{1}\right)+\gamma} .
$$

Proof. It is clear that the collection of balls $\mathcal{B}$ constructed above has the properties (i) and (ii) for $N$ sufficiently large (the estimate on the distance between the centres of the balls in $\mathcal{B}$ follows from (10) and (14)). The estimate for $|\mathcal{B}|$ in (iii) follows from (15) and the definition of $\mathcal{B}$, while the estimate for $X(N)$ follows from (3) and (7). We now prove (iv).

For any $[\mathbf{q}] \in[\widetilde{Q}(N)]$ and any pair $(\mathbf{q}, \mathbf{t}) \in S([\mathbf{q}])$, let $\mathcal{B}(\mathbf{q}, \mathbf{t})$ be the set of all balls $B \in \mathcal{B}([\mathbf{q}])$ which belong to any collection $\mathcal{B}\left(B^{0}\right)$ for which 
the centre of $B^{0}$ lies on the plane $H(\mathbf{q}, \mathbf{t})$ (i.e., $\mathcal{B}(\mathbf{q}, \mathbf{t})$ is the set of all balls $B \in \mathcal{B}(\mathbf{q}, \mathbf{t})$ which lie "close" to the plane $H(\mathbf{q}, \mathbf{t}))$. It follows from the above constructions that if $(\mathbf{q}, \mathbf{t}) \neq\left(\mathbf{q}^{\prime}, \mathbf{t}^{\prime}\right)$ and $B \in \mathcal{B}(\mathbf{q}, \mathbf{t}), B^{\prime} \in \mathcal{B}\left(\mathbf{q}^{\prime}, \mathbf{t}^{\prime}\right)$ then their centres $Z, Z^{\prime}$ satisfy (14).

Now suppose that $d(I)$ satisfies the inequalities in case (a) for some $k, 1 \leq k \leq m-1$. We begin by estimating the number $h(\mathbf{q}, \mathbf{t})$ of balls $B \in \mathcal{B}(\mathbf{q}, \mathbf{t})$ which can intersect $I$. Since the balls $B \in \mathcal{B}(\mathbf{q}, \mathbf{t})$ have diameters $n^{-1}(2 N)^{-\left(1+\tau_{1}\right)}$, their centres are a distance at least $N^{-\left(1+\tau_{1}\right)}$ apart, and they all lie "close" to the $m(n-1)$-dimensional plane $H(\mathbf{q}, \mathbf{t})$, it follows from the geometry of the situation and the construction of the collection $\mathcal{B}(\mathbf{q}, \mathbf{t})$ that the number $h(\mathbf{q}, \mathbf{t})$ of balls $B \in \mathcal{B}(\mathbf{q}, \mathbf{t})$ which can intersect $I$ satisfies

$$
\begin{aligned}
h(\mathbf{q}, \mathbf{t}) & \ll\left(\frac{d(I)}{N^{-\left(1+\tau_{1}\right)}}\right)^{n(m-k)} \prod_{i=1}^{k} N^{\tau_{1}-\tau_{i}}\left(\frac{d(I)}{N^{-\left(1+\tau_{1}\right)}}\right)^{n-1} \\
& \leq d(I)^{m n-k} N^{(m n-k)\left(1+\tau_{1}\right)+\sum_{i=1}^{k}\left(\tau_{1}-\tau_{i}\right)} .
\end{aligned}
$$

Now, if $k<K$ then by $(\tau 2)$, (14) and the above construction, if $N$ is sufficiently large, $I$ can intersect balls from at most one collection $\mathcal{B}(\mathbf{q}, \mathbf{t})$ with $(\mathbf{q}, \mathbf{t}) \in \bigcup_{[\mathbf{q}] \in[\widetilde{Q}(N)]} S([\mathbf{q}])$. Thus (16) follows from (21). Next, if $k>K$ then by $(\tau 1),(\tau 2),(14)$ and the above construction, if $N$ is sufficiently large the number of collections $\mathcal{B}(\mathbf{q}, \mathbf{t})$ which contain balls intersecting $I$ is

$$
\ll \prod_{i=1}^{k} \frac{d(I)}{N^{-1-\widetilde{\tau}_{i}-\delta / m}}=d(I)^{k} N^{k+\sum_{i=1}^{k} \widetilde{\tau}_{i}+k \delta / m} .
$$

Therefore, in this case it follows from (21) and (22) that the total number of balls intersecting $I$ is $\ll d(I)^{m n} N^{\zeta}$, where

$$
\begin{aligned}
\zeta= & k+\sum_{i=1}^{k} \widetilde{\tau}_{i}+k \delta / m+(m n-k)\left(1+\tau_{1}\right)+\sum_{i=1}^{k}\left(\tau_{1}-\tau_{i}\right) \\
= & m(n-1)\left(1+\tau_{1}\right)+m+\sum_{i=1}^{m} \widetilde{\tau}_{i}-\sum_{i=k+1}^{m} \widetilde{\tau}_{i}+\sum_{i=1}^{m}\left(\tau_{1}-\tau_{i}\right) \\
& +\sum_{i=k+1}^{m} \tau_{i}+k \delta / m \\
= & m(n-1)\left(1+\tau_{1}\right)+m+\nu+\gamma+\sum_{i=k+1}^{m}\left(\tau_{i}-\widetilde{\tau}_{i}\right)+(k \delta) / m \\
\leq & m(n-1)\left(1+\tau_{1}\right)+m+\gamma+\nu+\delta
\end{aligned}
$$

(using $(\tau 2)$ and $(\tau 3)$ ). This proves (18). Finally (in case (a)), suppose that $k=K$. Then, using the above arguments, if $d(I)<N^{-1-\widetilde{\tau}_{K}-\delta / m}$ we obtain 
the estimate (16), while if $d(I) \geq N^{-1-\widetilde{\tau}_{K}-\delta / m}$ we obtain the estimate (18). Adding these estimates yields (17), which completes the proof of case (a).

Next, consider case (b). For a fixed equivalence class $[\mathbf{q}] \in[\widetilde{Q}(N)]$, it follows from (6) that the number of collections $\mathcal{B}(\mathbf{q}, \mathbf{t})$ with $(\mathbf{q}, \mathbf{t}) \in S([\mathbf{q}])$, which have at least one ball intersecting the set $I$, is

$$
\ll \prod_{i=1}^{m} \frac{d(I)}{N^{-1-\widetilde{\tau}_{i}+\alpha / m-\delta / m}}=d(I)^{m} N^{m+\nu-\alpha+\delta},
$$

and the number of balls $B$ in each such collection $\mathcal{B}(\mathbf{q}, \mathbf{t})$ is

$$
\ll\left(\frac{d(I)}{N^{-\left(1+\tau_{1}\right)}}\right)^{m(n-1)} \prod_{i=1}^{m} N^{\tau_{1}-\tau_{i}}=d(I)^{m(n-1)} N^{m(n-1)\left(1+\tau_{1}\right)+\gamma} .
$$

Hence the number of balls corresponding to a single equivalence class which intersect $I$ is

$$
\ll d(I)^{m n} N^{m+\nu-\alpha+\delta+m(n-1)\left(1+\tau_{1}\right)+\gamma} .
$$

The number of possible equivalence classes is $\ll N^{\alpha-\delta}$ which, together with the above estimate, gives (19).

Finally, in case (c) it follows from (iv) of Lemma 2.5 that the number of collections $\mathcal{B}(\mathbf{q}, \mathbf{t})$ with $(\mathbf{q}, \mathbf{t}) \in S([\mathbf{q}])$, which have at least one ball intersecting the set $I$ is $\ll d(I)^{m} \chi([\mathbf{q}])$. Using the estimate (23) for the number of balls in each such collection and summing over the set of equivalence classes $[\mathbf{q}] \in[\widetilde{Q}(N)]$ yields $(20)$. This completes the proof of Lemma 2.7.

Now, it will be shown that if $\delta>0$ is sufficiently small then we have $\operatorname{dim} W_{Q}(m, n ; \boldsymbol{\tau}) \geq \varrho:=D_{Q}(m, n ; \boldsymbol{\tau})-4 \delta$. On letting $\delta \rightarrow 0$ this yields the required lower bound for $\operatorname{dim} W_{Q}(m, n ; \boldsymbol{\tau})$, which will complete the proof, subject to the additional conditions imposed above.

Choose $N_{0}>0$ sufficiently large that

$$
4 c_{3} N_{0}^{-(\sigma-\nu)-\delta\left(1+\tau_{1}\right)} \leq c_{2}
$$

(this is possible since $\sigma>\nu$ ). Let $\mathcal{F}$ be any countable family of sets $I$ in $\mathbb{R}^{n}$ of positive diameter $d(I) \leq \frac{1}{2} n^{-1}\left(2 N_{0}\right)^{-\left(1+\tau_{1}\right)}$ with

$$
V_{\varrho}(\mathcal{F})=\sum_{I \in \mathcal{F}} d(I)^{\varrho}<1 .
$$

We will show that the family $\mathcal{F}$ cannot cover the set $W_{Q}(m, n ; \boldsymbol{\tau}) \cap U$ and hence, by definition, $m_{\varrho}\left(W_{Q}(m, n ; \boldsymbol{\tau})\right)>0$, which proves $\operatorname{dim} W_{Q}(m, n ; \boldsymbol{\tau})$ $\geq \varrho$. To do this we construct a sequence of sets $U \supset J_{0} \supset J_{1} \supset \ldots$, where $J_{j} \subset \mathbb{R}^{m n}$ is the union of $M_{j}>0$ pairwise disjoint balls and integers $N_{0}<N_{1}<\ldots$, such that for $j \geq 1$, the following conditions are satisfied: 
(i) ${ }_{j} J_{j}$ intersects no $I \in \mathcal{F}$ with $d(I)>\frac{1}{2} n^{-1}\left(2 N_{j}\right)^{-\left(1+\tau_{1}\right)}$;

(ii) ${ }_{j}$ each ball of $J_{j}$ has diameter $n^{-1}\left(2 N_{j}\right)^{-\left(1+\tau_{1}\right)}$ and their centres are at least a distance $4 N_{j}^{-\left(1+\tau_{1}\right)}$ apart;

(iii) $)_{j}$ if $X \in J_{j}$, there is a $\mathbf{q} \in \widetilde{Q}\left(N_{j}\right)$ such that $\left\|\mathbf{x}_{i} \cdot \mathbf{q}\right\|<|\mathbf{q}|^{-\tau_{i}}$, for $i=1, \ldots, m$;

(iv) ${ }_{j} M_{j} \geq 4 c_{3} c_{2}^{-1} 2^{m n\left(1+\tau_{1}\right)} N_{j}^{-(\sigma-\nu)+m n\left(1+\tau_{1}\right)-\delta\left(1+\tau_{1}\right)}$ (we suppose that $\delta$ is sufficiently small that the exponent of $N_{j}$ here is positive).

Supposing that such sequences exist, let

$$
J_{\infty}=\bigcap_{j=0}^{\infty} J_{j} .
$$

Since the sequence $J_{j}, j=0,1, \ldots$, is a decreasing sequence of non-empty closed bounded sets in $\mathbb{R}^{m n}, J_{\infty}$ is non-empty. By (i) ${ }_{j}, J_{\infty}$ does not intersect any set $I \in \mathcal{F}$, while by (iii) ${ }_{j}, J_{\infty} \subset W_{Q}(m, n ; \boldsymbol{\tau})$. Thus, $\mathcal{F}$ does not cover $W_{Q}(m, n ; \boldsymbol{\tau})$.

The construction is by induction. Let $J_{0}$ be the ball of diameter 1 and centre $\left(\frac{1}{2}, \ldots, \frac{1}{2}\right)$, and let $N_{0}$ be as above. Now suppose that $J_{0}, J_{1}, \ldots, J_{j-1}$, $N_{0}, N_{1}, \ldots, N_{j-1}$ have already been constructed satisfying the above conditions, for some $j \geq 1$. We will construct $J_{j}$ and $N_{j}$. Let $D$ be a ball of $J_{j-1}$ and let $C=\frac{1}{4} D$. Applying Lemma 2.7 to $C$ we choose $N_{j}=N$ such that $N_{j}^{-1+\delta}<n^{-1}\left(2 N_{j-1}\right)^{-\left(1+\tau_{1}\right)}$, and we obtain the corresponding collection of balls $\mathcal{B}=\mathcal{B}(D)$. Let

$$
\mathcal{G}_{j}=\bigcup_{D \in J_{j-1}} \mathcal{B}(D)
$$

and let

$$
\begin{aligned}
\mathcal{F}_{j}^{1, k} & =\left\{I \in \mathcal{F}: N_{j}^{-\left(1+\tau_{k}\right)}<d(I) \leq N_{j}^{-\left(1+\tau_{k+1}\right)}\right\}, \quad k=1, \ldots, m-1, \\
\mathcal{F}_{j}^{2} & =\left\{I \in \mathcal{F}: N_{j}^{-\left(1+\tau_{m}\right)}<d(I) \leq N_{j}^{-1+\delta}\right\}, \\
\mathcal{F}_{j}^{3} & =\left\{I \in \mathcal{F}: N_{j}^{-1+\delta}<d(I) \leq N_{j-1}^{-\left(1+\tau_{1}\right)}\right\} .
\end{aligned}
$$

Taking $\mathcal{H}_{j}$ to be the set of balls in $\mathcal{G}_{j}$ which intersect a set $I \in \bigcup_{k} \mathcal{F}_{j}^{1, k} \cup$ $\mathcal{F}_{j}^{2} \cup \mathcal{F}_{j}^{3}$, we define $J_{j}$ to be the union of the balls in the collection $\mathcal{G}_{j} \backslash \mathcal{H}_{j}$. Thus, we have $J_{j} \subset J_{j-1}$ and (i) ${ }_{j}$ holds (because $d(I) \leq \frac{1}{2} n^{-1}\left(2 N_{0}\right)^{-\left(1+\tau_{1}\right)}$, $I \in \mathcal{F}$, if $j=1$, and because of (i) ${ }_{j-1}$ if $j>1$ ). Also, (ii) ${ }_{j}$ and (iii) ${ }_{j}$ follow from (i) and (ii) of Lemma 2.7. It remains to consider (iv) ${ }_{j}$.

If $I \in \bigcup_{k} \mathcal{F}_{j}^{1, k} \cup \mathcal{F}_{j}^{2} \cup \mathcal{F}_{j}^{3}$, then $I$ cannot intersect balls in $\mathcal{B}(D)$ for two distinct balls $D \in J_{j-1}$ (because of (ii) $j_{-1}$, if $j>1$ ). Therefore, by part (iv) of Lemma 2.7, 


$$
\begin{aligned}
c_{3}^{-1}\left|\mathcal{H}_{j}\right| \leq & \sum_{k=1}^{K} \sum_{I \in \mathcal{F}_{j}^{1, k}} d(I)^{m n-k} N_{j}^{(m n-k)\left(1+\tau_{1}\right)+\sum_{i=1}^{k}\left(\tau_{1}-\tau_{i}\right)} \\
& +\sum_{k=K}^{m-1} \sum_{I \in \mathcal{F}_{j}^{1, k}} d(I)^{m n} N_{j}^{m(n-1)\left(1+\tau_{1}\right)+m+\nu+\gamma+\delta} \\
& +\sum_{I \in \mathcal{F}_{j}^{2}} d(I)^{m n} N_{j}^{m(n-1)\left(1+\tau_{1}\right)+m+\nu+\gamma} \\
& +\sum_{I \in \mathcal{F}_{j}^{3}} d(I)^{m n} X\left(N_{j}\right) N_{j}^{m(n-1)\left(1+\tau_{1}\right)+\gamma}
\end{aligned}
$$

We now estimate the various sums in (26). First we consider the integers $k$ such that $1 \leq k \leq K$, and suppose that $m n-k-\varrho \leq 0$. Then, by the definition of $\mathcal{F}_{j}^{1, k}$, we have

$$
d(I)^{m n-k}=d(I)^{\varrho} d(I)^{m n-k-\varrho} \leq d(I)^{\varrho} N_{j}^{-(m n-k-\varrho)\left(1+\tau_{k}\right)},
$$

and so, using (25), we obtain

$$
\sum_{I \in \mathcal{F}_{j}^{1, k}} d(I)^{m n-k} N_{j}^{(m n-k)\left(1+\tau_{1}\right)+\sum_{i=1}^{k}\left(\tau_{1}-\tau_{i}\right)} \ll N_{j}^{\zeta},
$$

where

$$
\zeta=-(m n-k-\varrho)\left(1+\tau_{k}\right)+(m n-k)\left(1+\tau_{1}\right)+\sum_{i=1}^{k}\left(\tau_{1}-\tau_{i}\right) .
$$

Now, by the definition of $D_{Q}(m, n ; \boldsymbol{\tau})$,

$$
\begin{aligned}
-(m n-k-\varrho)\left(1+\tau_{k}\right) \leq & -(m-k)\left(1+\tau_{k}\right)+m+\nu \\
& +\sum_{i=k}^{m}\left(\tau_{k}-\tau_{i}\right)-4 \delta\left(1+\tau_{k}\right) \\
= & k+\nu-\sum_{i=k+1}^{m} \tau_{i}-4 \delta\left(1+\tau_{k}\right),
\end{aligned}
$$

so

$$
\zeta \leq m(n-1)\left(1+\tau_{1}\right)+m+\nu+\gamma-4 \delta\left(1+\tau_{k}\right) .
$$

If $m n-k-\varrho>0$ similar calculations yield

$$
\zeta=-(m n-k-\varrho)\left(1+\tau_{k+1}\right)+(m n-k)\left(1+\tau_{1}\right)+\sum_{i=1}^{k}\left(\tau_{1}-\tau_{i}\right),
$$

and 


$$
\begin{aligned}
& -(m n-k-\varrho)\left(1+\tau_{k+1}\right) \\
& \quad \leq-(m-k)\left(1+\tau_{k+1}\right)+m+\nu+\sum_{i=k+1}^{m}\left(\tau_{k+1}-\tau_{i}\right)-4 \delta\left(1+\tau_{k+1}\right) \\
& \quad=k+\nu-\sum_{i=k+1}^{m} \tau_{i}-4 \delta\left(1+\tau_{k+1}\right),
\end{aligned}
$$

SO

$$
\zeta \leq m(n-1)\left(1+\tau_{1}\right)+m+\nu+\gamma-4 \delta\left(1+\tau_{k+1}\right) .
$$

Next we consider $k$ such that $K \leq k \leq m-1$. In this case we use

$$
m n-\varrho \geq \frac{m \tau_{1}-\nu-\sum_{i=1}^{m}\left(\tau_{1}-\tau_{i}\right)}{1+\tau_{1}}+4 \delta=\frac{\sigma-\nu}{1+\tau_{1}}+4 \delta>4 \delta>0
$$

(since $\sigma>\nu$ ), to obtain the estimate

$$
\sum_{I \in \mathcal{F}_{j}^{1, k}} d(I)^{m n} N_{j}^{m(n-1)\left(1+\tau_{1}\right)+m+\nu+\gamma+\delta} \ll N_{j}^{\zeta},
$$

where

$$
\begin{aligned}
\zeta & =-(m n-\varrho)\left(1+\tau_{k+1}\right)+m(n-1)\left(1+\tau_{1}\right)+m+\nu+\gamma+\delta \\
& <m(n-1)\left(1+\tau_{1}\right)+m+\nu+\gamma-3 \delta
\end{aligned}
$$

for $\delta$ sufficiently small.

For the summation over $\mathcal{F}_{j}^{2}$ in (26) we again use (27) to obtain a similar estimate with

$$
\begin{aligned}
\zeta & =-(m n-\varrho)(1-\delta)+m(n-1)\left(1+\tau_{1}\right)+m+\nu+\gamma+\delta \\
& <m(n-1)\left(1+\tau_{1}\right)+m+\nu+\gamma-3 \delta
\end{aligned}
$$

for $\delta$ sufficiently small.

Finally, for the summation over $\mathcal{F}_{j}^{3}$ in (26) we obtain (using (27))

$$
\begin{aligned}
\sum_{I \in \mathcal{F}_{j}^{3}} d(I)^{m n} X\left(N_{j}\right) N_{j}^{m(n-1)\left(1+\tau_{1}\right)+\gamma} & \\
& \leq N_{j-1}^{-(m n-\varrho)\left(1+\tau_{1}\right)} X\left(N_{j}\right) N_{j}^{m(n-1)\left(1+\tau_{1}\right)+\gamma} \\
& \leq N_{j-1}^{-(\sigma-\nu)-\delta\left(1+\tau_{1}\right)} X\left(N_{j}\right) N_{j}^{m(n-1)\left(1+\tau_{1}\right)+\gamma} .
\end{aligned}
$$

Combining the above estimates, we obtain

$$
\left|\mathcal{H}_{j}\right| \leq 2 c_{3} N_{j-1}^{-(\sigma-\nu)-\delta\left(1+\tau_{1}\right)} X\left(N_{j}\right) N_{j}^{m(n-1)\left(1+\tau_{1}\right)+\gamma},
$$

for sufficiently large $N_{j}$ (using the estimate $X(N) \gg N^{m+\nu-3 \delta / 2}$ in Lemma 2.7). 
Now suppose that $j=1$. By (iii) of Lemma 2.7 (with $d(C)=1$ ), together with (24) and (28),

$$
\left|\mathcal{G}_{1}\right| \geq c_{2} X\left(N_{1}\right) N_{1}^{m(n-1)\left(1+\tau_{1}\right)+\gamma} \geq 2\left|\mathcal{H}_{1}\right| .
$$

Hence,

$$
M_{1} \geq\left|\mathcal{G}_{1}\right|-\left|\mathcal{H}_{1}\right| \geq c_{2} 2^{-1} X\left(N_{1}\right) N_{1}^{m(n-1)\left(1+\tau_{1}\right)+\gamma},
$$

so (iv) ${ }_{1}$ holds for sufficiently large $N_{1}$.

Next suppose that $j>1$. Then, by (iii) of Lemma 2.7 , (ii) $)_{j-1}$, (iv) $)_{j-1}$ and (28),

$$
\begin{aligned}
\left|\mathcal{G}_{j}\right| & \geq M_{j-1} c_{2}\left(2 N_{j-1}\right)^{-m n\left(1+\tau_{1}\right)} X\left(N_{j}\right) N_{j}^{m(n-1)\left(1+\tau_{1}\right)+\gamma} \\
& \geq 4 c_{3} N_{j-1}^{-(\sigma-\nu)-\delta\left(1+\tau_{1}\right)} X\left(N_{j}\right) N_{j}^{m(n-1)\left(1+\tau_{1}\right)+\gamma} \geq 2\left|\mathcal{H}_{j}\right| .
\end{aligned}
$$

Thus, $M_{j} \geq\left|\mathcal{G}_{j}\right|-\left|\mathcal{H}_{j}\right| \geq \frac{1}{2}\left|\mathcal{G}_{j}\right|$, and it follows from (29) that (iv) ${ }_{j}$ holds for sufficiently large $N_{j}$ if $\delta$ is sufficiently small. This completes the proof of the theorem under the various particular assumptions made in the course of the argument, viz., $\nu>\alpha>0$ and $\sigma>\nu$.

We now remove these assumptions. Firstly, we note that the cases when $\nu=\alpha>0$ and when $\nu=0$ (with $\sigma>\nu$ ), can be dealt with by a similar method to that described in the final paragraph of [10]. Next, when $\sigma=\nu$ (for any $\nu \geq 0$ ) the estimate $\operatorname{dim} W_{Q}(m, n ; \boldsymbol{\tau}) \geq D_{Q}(m, n ; \boldsymbol{\tau})$ follows from the result just proved by using the continuity argument following Lemma 1 in [11] (elements $\tau \in G$ have $\sigma>\nu$, but any $\tau$ for which $\sigma=\nu$ lies on the boundary of $G$ ).

Now suppose that $\sigma \leq \nu$. Then, for each $k$ with $1 \leq k \leq m$,

$$
\begin{aligned}
\frac{m+\nu+\sum_{i=k}^{m}\left(\tau_{k}-\tau_{i}\right)}{1+\tau_{k}} & \geq \frac{m+\sum_{i=1}^{m} \tau_{i}+\sum_{i=k}^{m}\left(\tau_{k}-\tau_{i}\right)}{1+\tau_{k}} \\
& =\frac{m+\sum_{i=1}^{k-1} \tau_{i}+\sum_{i=k}^{m} \tau_{k}}{1+\tau_{k}} \\
& \geq \frac{m+\sum_{i=1}^{m} \tau_{k}}{1+\tau_{k}}=m,
\end{aligned}
$$

and hence, by the definition, $D_{Q}(m, n ; \boldsymbol{\tau}) \geq m n$. Furthermore, if $\sigma=\nu$ then for $k=1$,

$$
\frac{m+\nu+\sum_{i=1}^{m}\left(\tau_{1}-\tau_{i}\right)}{1+\tau_{1}}=\frac{m+\sum_{i=1}^{m} \tau_{1}}{1+\tau_{1}}=m
$$

so, together with the previous estimates, this shows that in this case $\operatorname{dim} W_{Q}(m, n ; \boldsymbol{\tau})=D_{Q}(m, n ; \boldsymbol{\tau})=m n$.

Now suppose that $\sigma<\nu$. Then, by increasing the components of the vector $\boldsymbol{\tau}$ appropriately, we can construct a vector $\overline{\boldsymbol{\tau}}$ such that $\sigma(\overline{\boldsymbol{\tau}})=\nu$, and hence, since $W_{Q}(m, n ; \overline{\boldsymbol{\tau}}) \subset W_{Q}(m, n ; \boldsymbol{\tau})$, the above result for the case $\sigma=\nu$ 
gives

$$
\operatorname{dim} W_{Q}(m, n ; \boldsymbol{\tau}) \geq \operatorname{dim} W_{Q}(m, n ; \bar{\tau})=m n,
$$

which finally completes the proof of the theorem.

Acknowledgements. H. Dickinson is supported by EPSRC grant GR/K56407.

\section{References}

[1] A. S. Besicovitch, Sets of fractional dimension (IV): On rational approximation to real numbers, J. London Math. Soc. 9 (1934), 126-131.

[2] I. Borosh and A. S. Fraenkel, A generalization of Jarnik's theorem on Diophantine approximations, Indag. Math. 34 (1972), 193-201.

[3] J. D. Bovey and M. M. Dodson, The Hausdorff dimension of systems of linear forms, Acta Arith. 45 (1986), 337-358.

[4] M. M. Dodson, Hausdorff dimension, lower order and Khintchine's theorem in metric Diophantine approximation, J. Reine Angew. Math. 432 (1992), 69-76.

[5] M. M. Dodson and J. A. G. Vickers, Exceptional sets in Kolmogorov-Arnol'dMoser theory, J. Phys. A 19 (1986), 349-374.

[6] I. R. Dombrovski, Simultaneous approximations of real numbers by algebraic numbers of bounded degree, Dokl. Akad. Nauk BSSR 33 (1989), 205-208.

[7] H. G. Eggleston, Sets of fractional dimension which occur in some problems in number theory, Proc. London Math. Soc. 54 (1952), 42-93.

[8] V. Jarník, Diophantische Approximationen und Hausdorffsches Mass, Mat. Sb. 36 (1929), 371-382.

[9] —, Über die simultanen Diophantischen Approximationen, Math. Z. 33 (1931), 503543.

[10] B. P. Rynne, The Hausdorff dimension of certain sets arising from Diophantine approximation by restricted sequences of integer vectors, Acta Arith. 61 (1992), 69-81.

[11] - Hausdorff dimension and generalized Diophantine approximation, Bull. London Math. Soc. 30 (1998), 365-376.

[12] V. G. Sprindžuk, Metric Theory of Diophantine Approximations (translated by R. A. Silverman), Winston, Washington, 1979.

Department of Mathematics

Heriot-Watt University

Edinburgh EH14 4AS, U.K.

E-mail: b.p.rynne@ma.hw.ac.uk
Department of Mathematics University of York York YO1 5DD, U.K. E-mail: hd3@york.ac.uk

Received on 22.12.1998

and in revised form on 11.10.1999 